\title{
Observation of a Neutrino Burst from the Supernova SN1987A
}

\author{
K. Hirata, ${ }^{(a)}$ T. Kajita, ${ }^{(a)}$ M. Koshiba, ${ }^{(a, b)}$ M. Nakahata, ${ }^{(b)}$ Y. Oyama, ${ }^{(b)}$ \\ N. Sato, ${ }^{(c)}$ A. Suzuki, ${ }^{(b)}$ M. Takita, ${ }^{(b)}$ and Y. Totsuka ${ }^{(a, c)}$ \\ University of Tokyo, Tokyo 113, Japan \\ T. Kifune and T. Suda \\ Institute for Cosmic Ray Research, University of Tokyo, Tokyo 118, Japan \\ K. Takahashi and T. Tanimori \\ National Laboratory for High Energy Physics (KEK), Ibaraki 305, Japan \\ K. Miyano and M. Yamada \\ Department of Physics, University of Niigata, Niigata 950-21, Japan
}

E. W. Beier, L. R. Feldscher, S. B. Kim, A. K. Mann, F. M. Newcomer, R. Van Berg, and W. Zhang Department of Physics, University of Pennsylvania, Philadelphia, Pennsylvania 19104

and

B. G. Cortez ${ }^{(\mathrm{d})}$

California Institute of Technology, Pasadena, California 91125

(Received 10 March 1987)

\begin{abstract}
A neutrino burst was observed in the Kamiokande II detector on 23 February 1987, 7:35:35 UT ( \pm 1 min) during a time interval of $13 \mathrm{sec}$. The signal consisted of eleven electron events of energy 7.5 to 36 $\mathrm{MeV}$, of which the first two point back to the Large Magellanic Cloud with angles $18^{\circ} \pm 18^{\circ}$ and $15^{\circ} \pm 27^{\circ}$.

PACS numbers: $97.60 . \mathrm{Bw}, 14.60 . \mathrm{Gh}, 95.85 . \mathrm{Sz}, 97.60 . \mathrm{Jd}$
\end{abstract}

Following the optical sighting on 24 February 1987 of the supernova ${ }^{1}$ now called SN1987A, a search was made of the data taken in the detector Kamiokande-II during the period from 16:09, 21 February 1987 to $07: 31,24$ February 1987. We report here the results of that search.

The Kamiokande II detector, directed primarily at nucleon decay and solar $-{ }^{8} \mathrm{~B}$-neutrino detection, has been operating since the beginning of 1986. It is described in detail elsewhere, ${ }^{2}$ but its salient features are shown schematically in Fig. 1. The inner detector fiducial volume containing 2140 tons of water ${ }^{3}$ is viewed by an array of 20-in.-diameter photomultiplier tubes (PMT's) on a $1 \times 1-\mathrm{m}^{2}$ lattice on the surface. The photocathode coverage amounts to $20 \%$ of the total inner surface. The attenuation length of the water for Cherenkov light is in excess of $45 \mathrm{~m}$. The inner detector is completely surrounded by a water Cherenkov counter of thickness $\geqq 1.4 \mathrm{~m}$ to ensure the containment of low-energy events. The outer counter also is an absorber of $\gamma$ rays from surrounding rock and a monitor of slow muons which do not produce Cherenkov light in the inner detector but may decay there.

Neutrinos of different flavors are detected through the scattering reaction $v e \rightarrow v e$. The kinematics of this reaction and the subsequent multiple scattering of the recoiling electron preserve knowledge of the incident neutrino

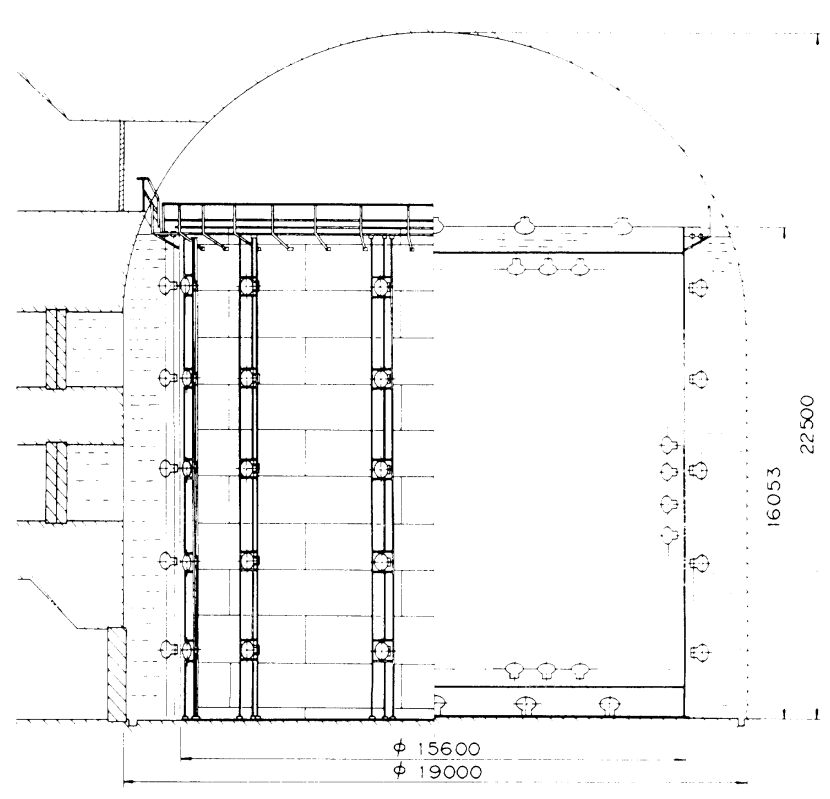

FIG. 1. Schematic view of the Kamiokande II detector. The inner detector contains 3000 tons of water of which 2140 tons are fiducial volume. It is viewed by 94820 -in.-diameter PMT's mounted on a 1-m grid on the inner surface. The outer (veto) counter surrounds the inner detector and is viewed by 123 PMT's. Dimensions in the figure are in millimeters. 
direction within approximately $28^{\circ} \mathrm{rms}$ at electron energies in the vicinity of $10 \mathrm{MeV}$. In addition, $\bar{v}_{e}$ are detected through the reaction $\bar{v}_{e} p^{+} \rightarrow e^{+} n$ on the free protons in the water with a cross section approximately 100 times larger than the cross section for $v_{e} e \rightarrow v_{e} e$ at $10 \mathrm{MeV}$. The reaction $\bar{v}_{e} p^{+} \rightarrow e^{+} n$ produces $e^{+}$essentially isotropically. The Cherenkov light of a $10-\mathrm{MeV}$ electron gives on average 26.3 hit PMT's $\left(N_{\text {hit }}\right)$ at $\frac{1}{3}$ photoelectron threshold. The energy calibration is obtained by observation of $\mu \rightarrow e$ decays and by use of the Compton-scattered electrons from $\gamma$ rays of energy up to $9 \mathrm{MeV}$ from $n+\mathrm{Ni}$ by use of a $\mathrm{Cf}$ neutron source.

The detector is triggered by 20 PMT discriminators firing within $100 \mathrm{nsec}$. The trigger dead time is approximately $50 \mathrm{nsec}$. Charge and time information for each channel above threshold is recorded for each trigger. The trigger accepts $8.5-\mathrm{MeV}$ electrons with $50 \%$ efficiency and 14-MeV electrons with $90 \%$ efficiency over the volume of the detector. ${ }^{3}$ The raw trigger rate is 0.60 $\mathrm{Hz}$ of which $0.37 \mathrm{~Hz}$ is cosmic-ray muons. The remaining $0.23 \mathrm{~Hz}$ is largely due to radioactive contamination in the water.

Reconstruction of the vertices of low-energy events is performed with an algorithm based on the time and position of hit PMT's. After the vertex is established, a separate fit is used to obtain the angle of the electron. The distribution of the events presented here is consistent with a uniform volume distribution.

The search for a neutrino burst from SN1987A was carried out on the data of run 1892, which, except for a pedestal run of $105 \mathrm{sec}$ duration every hour, continuously covered the period from 16:09, 21 February 1987 to 0731, 24 February 1987, in Japanese Standard Time (JST), which is UT plus $9 \mathrm{~h}$. Events satisfying the following three criteria were selected: (1) The total number of photoelectrons per event in the inner detector had to be less than 170 , corresponding to a $50-\mathrm{MeV}$ electron; (2) the total number of photoelectrons in the outer detector had to be less than 30 , ensuring event containment; and (3) the time interval from the preceding event had to be longer than $20 \mu \mathrm{sec}$, to exclude electrons from muon decay.

The short-time correlation of these low-energy contained events was investigated and the event sequence as shown in Fig. 2 was observed at 16:35:35 JST (7:35:35 UT) of 23 February 1987. In Fig. 2 we show the time sequence of all low-energy events (solid lines) and all cosmic-ray muon events (dashed lines) in the given interval. The event sequence during 0 to $2 \mathrm{sec}$ is shown expanded in the upper right corner. The properties of the events in the burst (numbered 1 to 12 in Fig. 2) are summarized in Table I. Event number 6 has $N_{\text {hit }}<20$ and has been excluded from the signal analysis. A scatter plot of event energy versus cosine of the angle between the measured electron direction and the direction of the Large Magellanic Cloud (LMC), known to contain SN1987A, is shown in Fig. 3. The zenith angle of the LMC was $109.7^{\circ}$ at the experimental site. It is seen that the earliest two events point back to LMC with angles $18^{\circ} \pm 18^{\circ}$ and $15^{\circ} \pm 27^{\circ}$. The angular distribution of

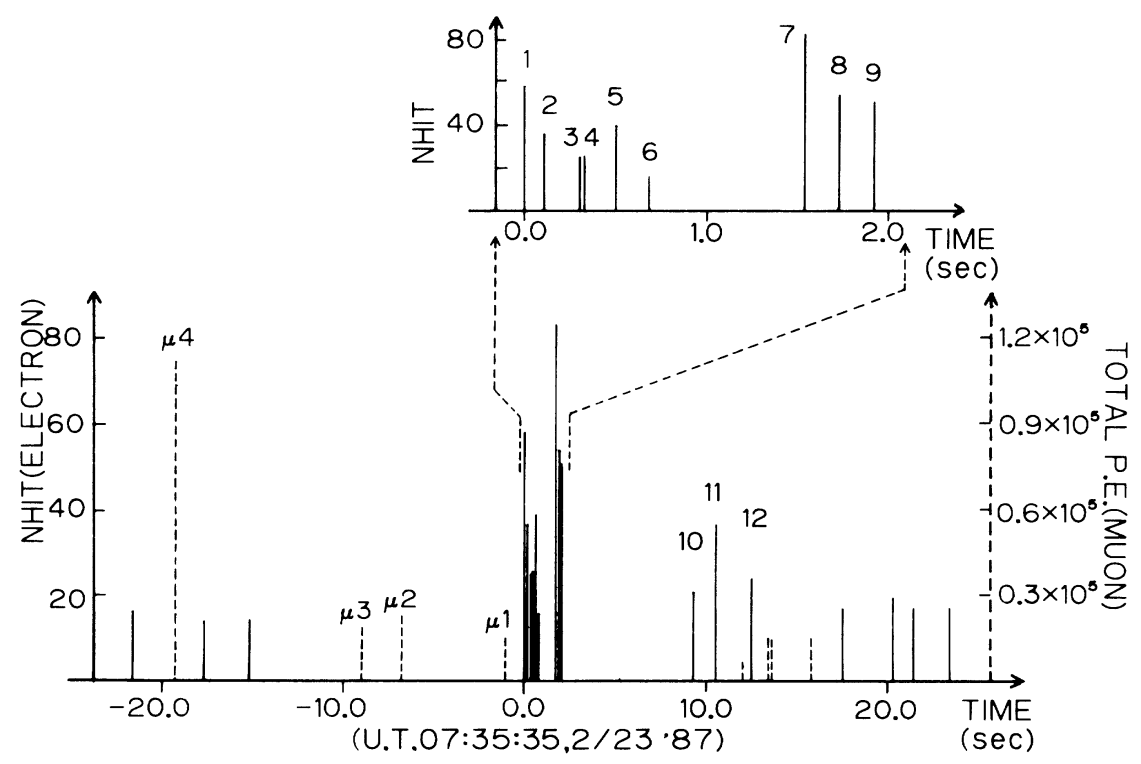

FIG. 2. The time sequence of events in a 45-sec interval centered on 07:35:35 UT, 23 February 1987. The vertical height of each line represents the relative energy of the event. Solid lines represent low-energy electron events in units of the number of hit PMT's, $N_{\text {hit }}$ (left-hand scale). Dashed lines represent muon events in units of the number of photoelectrons (right-hand scale). Events $\mu 1-\mu 4$ are muon events which precede the electron burst at time zero. The upper right figure is the $0-2$-sec time interval on an expanded scale. 
TABLE I. Measured properties of the twelve electron events detected in the neutrino burst. The electron angle in the last column is relative to the direction of SN1987A. The errors on electron energies and angles are one-standard-deviation Gaussian errors.

\begin{tabular}{ccccr}
\hline \hline $\begin{array}{c}\text { Event } \\
\text { number }\end{array}$ & $\begin{array}{c}\text { Event } \\
\text { time } \\
(\mathrm{sec})\end{array}$ & $\begin{array}{c}\text { Number } \\
\text { of PMT's } \\
\left(N_{\text {hit }}\right)\end{array}$ & $\begin{array}{c}\text { Electron } \\
\text { energy } \\
(\mathrm{MeV})\end{array}$ & $\begin{array}{r}\text { Electron } \\
\text { angle } \\
\text { (degrees) }\end{array}$ \\
\hline 1 & 0 & 58 & $20.0 \pm 2.9$ & $18 \pm 18$ \\
2 & 0.107 & 36 & $13.5 \pm 3.2$ & $15 \pm 27$ \\
3 & 0.303 & 25 & $7.5 \pm 2.0$ & $108 \pm 32$ \\
4 & 0.324 & 26 & $9.2 \pm 2.7$ & $70 \pm 30$ \\
5 & 0.507 & 39 & $12.8 \pm 2.9$ & $135 \pm 23$ \\
6 & 0.686 & 16 & $6.3 \pm 1.7$ & $68 \pm 77$ \\
7 & 1.541 & 83 & $35.4 \pm 8.0$ & $32 \pm 16$ \\
8 & 1.728 & 54 & $21.0 \pm 4.2$ & $30 \pm 18$ \\
9 & 1.915 & 51 & $19.8 \pm 3.2$ & $38 \pm 22$ \\
10 & 9.219 & 21 & $8.6 \pm 2.7$ & $122 \pm 30$ \\
11 & 10.433 & 37 & $13.0 \pm 2.6$ & $49 \pm 26$ \\
12 & 12.439 & 24 & $8.9 \pm 1.9$ & $91 \pm 39$ \\
\hline \hline
\end{tabular}

the remainder of the events is consistent with isotropy.

In performing the search of the data of 16:09, 21 February 1987 to $07: 31$, 24 February 1987 JST, the data were divided into successive 10 -sec intervals. Binning problems were avoided by offsetting each 10 -sec interval 10 times by an additional second. A search was also made on a larger data sample of 42.9 days, 9 January 1987-25 February 1987, and no other burst candidates were found, where a burst candidate was defined as an event multiplicity $\geq 4$ per $10 \mathrm{sec}$ with $N_{\text {hit }}$ per event $\geq 30$. From the extended period it was determined that the number of events with $N_{\text {hit }} \geq 30$ per $10 \mathrm{sec}$ was well described by a Poisson distribution of mean $\bar{n}=0.0121$ up to multiplicity 3 , excepting only the burst of multiplicity 6 shown in Table I. For a reduced threshold of $N_{\text {hit }}$ per event $\geq 20$ per $10 \mathrm{sec}$, a Poisson distribution also obtained with $\bar{n}=0.219$ up to multiplicity 4 , the only exception being the burst (of multiplicity 9) described above. Accordingly, the rate of occurrence of 6 events per $10 \mathrm{sec}$ with $N_{\text {hit }} \geq 30$, or 9 events per $10 \mathrm{sec}$ with $N_{\text {hit }} \geq 20$, due to a statistical fluctuation is less than one per $7 \times 10^{7} \mathrm{yr}$ or less than one per $1 \times 10^{5} \mathrm{yr}$, respectively.

The only background process that might conceivably give rise to a burst of events in a short interval of time would be the production of an energetic nuclear cascade by an incident cosmic-ray muon. The characteristics of such events have been studied in detail previously as the spallation background for solar- ${ }^{8} \mathrm{~B}$-neutrino events. ${ }^{4}$ The relative total rate of spallation leading to one or more low-energy electron events is less than $10^{-3}$ per incident muon. The measured multiplicity distribution of low-energy electron events following an incident muon in time yields a probability of multiplicity $\geq 3$ of $3 \times 10^{-3}$. The low-energy electron-event background from spalla-
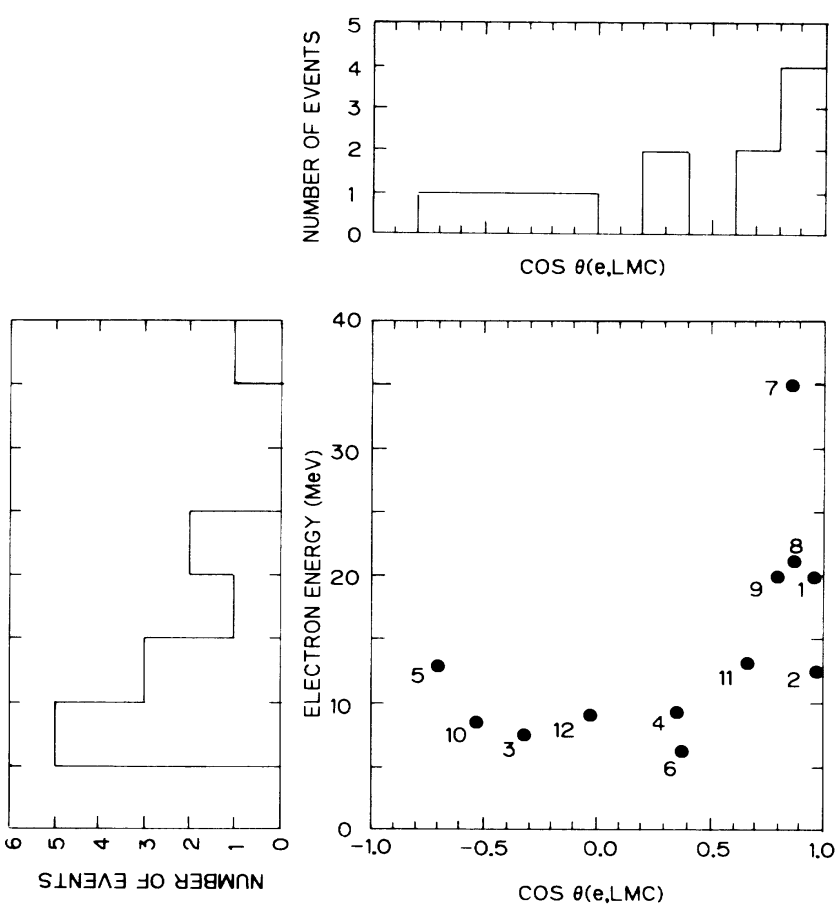

FIG. 3. Scatter plot of the detected electron energy in megaelectronvolts and the cosine of the angle between the measured electron direction and the direction of the Large Magellanic Cloud. The number to the left of each entry is the time-sequential event number from Table I. The two projections of the scatter plot are also displayed.

tion has the following principal properties: (1) It exhibits an exponential time structure which reflects the known lifetimes of the radioisotope fragments from ${ }^{16} \mathrm{O}$, specifically, an (18 \pm 1.2$)-m s e c$ component from ${ }^{12} \mathrm{~N}$ and ${ }^{12} \mathrm{~B}$, and also a component with a longer exponential time structure of $1.2 \pm 0.5 \mathrm{sec}$, with relative rates $2: 1$, respectively; and (2) the resultant $\beta$-decay electrons with observed energies above $15 \mathrm{MeV}$ occur with less than $4 \%$ probability.

Consequently, the overall probability that any of the muons, $\mu 1$ to $\mu 4$, was the progenitor of the event burst in Table $I$ is extremely low, much less than $10^{-3} \times 3$ $\times 10^{-3} \times(0.04)^{4}$, where the last factor follows from taking the four events (Nos. 1, 7, 8, and 9) in Table I with $E_{e}-1 \sigma>15 \mathrm{MeV}$. Note that the probability of 8 $\times 10^{-12}$ does not include factors from either the details of the internal time structure of the data in Table I, or the time separation of the entire burst from any of the preceding muons, or the geometrical correlation of the low-energy electrons from spallation with the muons that produced them.

We conclude that the event burst at 7:35:35 UT, 23 February 1987, displayed in Fig. 2 and Table I, is a genuine neutrino burst. This is the only such burst found by us during the period from 9 January to 25 February 
$1987 .^{5}$ We therefore associate it with SN1987A. This association is supported by the time structure of the events in the burst, their energy distribution, and uniform volume distribution. Additional support is provided by the correlation in angle of the first two observed events with the direction to SN1987A. The event burst occurred roughly $18 \mathrm{~h}$ prior to the first optical sighting. ${ }^{1}$ Correcting for energy-dependent detection efficiency, and assuming that nine of the twelve events are due to $\bar{v}_{e} p^{+} \rightarrow e^{+} n$, we obtain an integral flux of $1.0 \times 10^{10} \bar{v}_{e}$ $\mathrm{cm}^{-2}$ for the burst, where the $\bar{v}_{e}$ energy (the observed electron energy plus $1.3 \mathrm{MeV}$ ) is above $8.8 \mathrm{MeV}$. This, in turn, leads to the $\bar{v}_{e}$ output of SN1987A of $8 \times 10^{52}$ ergs for an assumed average energy of $15 \mathrm{MeV}$.

This observation is the first direct observation in neutrino astronomy, and coincides remarkably well with the current model of supernova collapse and neutron-star formation. ${ }^{6}$ In that model an aged, massive star, having exhausted its nuclear fuel, undergoes a supernova explosion. In supernovae of Type II almost all of the gravitational binding energy of the resultant neutron star, $\sim 3 \times 10^{53} \mathrm{ergs}$, is radiated within a few seconds in the form of $10^{58}$ neutrinos of all flavors with average energy in the vicinity of 10-15 MeV.

The impact on elementary-particle physics and the properties of neutrinos is direct. The lifetimes of $v_{e}$ and $\bar{v}_{e}$ must be greater than about $10^{5} \mathrm{yr} / \gamma$, where $\gamma$ is the ratio of neutrino energy to neutrino mass, thus ruling out neutrino decay as an explanation of the solar-neutrino puzzle. Neutrino mass and mixing information, and limits on the magnitudes of electromagnetic properties, are more problematical, and require additional study.

We gratefully acknowledge the cooperation of the Kamioka Mining and Smelting Company. This work was supported by the Japanese Ministry of Education, Science, and Culture, by the United States Department of Energy, and by the University of Pennsylvania Research Fund.

\footnotetext{
(a) International Center for Elementary Particle Physics.

(b) Department of Physics.

(c) Department of Astronomy.

(d) Present address: AT\&T Bell Laboratories, Holmdel, New Jersey 07922.
}

1I. Shelton, International Astronomical Union (IAU) Circular No. 4316. Note also that R. H. McNaught subsequently communicated visual magnitude of 6.0 on 23.44 UT February. The first confirmed observation of optical brightening was by G. Garradd, 23.44 February (IAU Circular No. 4316). The last confirmed evidence of no optical brightening was by Shelton, 23.059-23.101 February (IAU Circular No. 4330). There was a possible observation of no optical brightening by A. Jones at 23.39 February (IAU Circular No. 4340).

${ }^{2}$ E. W. Beier, in Proceedings of Seventh Workshop on Grand Unification, Toyama, Japan, April 1986 (to be published).

${ }^{3}$ This is for the entire volume inside the PMT array. The detection efficiency for a fiducial volume of 780 tons, $2 \mathrm{~m}$ inside the PMT array is $90 \%$ at $10 \mathrm{MeV}$ and $50 \%$ at $7.6 \mathrm{MeV}$.

${ }^{4} \mathrm{~A}$. Suzuki, in Proceedings of the Twelfth International Conference on Neutrino Physics and Astrophysics, June, 1986, Sendai, Japan, edited by T. Kitagaki (World Scientific, Singapore, 1986), p. 306.

${ }^{5}$ There is a claim of observation of a neutrino burst of five events of energy $\geqq 7 \mathrm{MeV}$ in $7 \mathrm{sec}, 23.124$ UT February (IAU Circular No. 4323), 02:52 UT (University of Torino Preprint), by Castagnoli et al., by use of a liquid scintillator detector of 90 tons effective mass, situated $5000 \mathrm{hg} / \mathrm{cm}^{2}$ under Mt. Blanc. If this were a real neutrino burst, our detector should have given about $(5 \pm \sqrt{5}) \times(2140 / 90) \times(1 / 1.29) \times 0.3=28 \pm 12$ events in $7 \mathrm{sec}$ at the same UT, where the second factor is the ratio of detector masses, the third factor is the ratio of free protons per unit mass in water and liquid scintillator, and the last factor is our detection efficiency for $7-\mathrm{MeV}$ electrons $\left(N_{\text {hit }} \geq 18\right)$. We searched carefully for such a burst in our data and found no sign of any burst at or around the claimed UT. In particular, during the 2-min interval centered at 02:52 UT 23 February 1987, the Poisson distribution of events per 10 sec with $N_{\text {hit }} \geq 20$ obtained, with $\bar{n}=0.219$. The maximum observed event multiplicity per $10 \mathrm{sec}$ satisfying $N_{\text {hit }} \geq 20$ in that 2 -min interval was 2 (one of which just satisfied $N_{\text {hit }} \geq 30$ ), which is expected from the Poisson distribution with a probability of 0.019 . The probability of finding by change one 10sec interval in that 2 -min period with multiplicity 2 is then $12 \times 0.019=0.23$. We conclude, as stated in the text, that no evidence for an event burst outside of statistics is present in our data at 02:52 UT $\pm 1 \min 23$ February 1987.

${ }^{6}$ S. A. Colgate and R. H. White, Astrophys. J. 143, 626 (1966); S. E. Woosley, J. Wilson, and R. Mayle, Astrophys. J. 302, 19 (1986); A. Burrows and J. M. Lattimer, Astrophys. J. 307, 178 (1986). For a review of the current understanding of the supernova phenomenon, see R. Mayle, Ph.D. thesis, University of California, Berkeley, 1985 (unpublished); S. E. Woosley and Thomas A. Weaver, Annu. Rev. Astron. Astrophys. 24, 205 (1986). 